\title{
Dissemination and Development of Wuqinxi in Singapore
}

\author{
Bin Zhang1*, Lei Ru², Hong Lin1, Jie Liang3, Chichou Lao33, Daiyin Peng1, Chunsong Cheng3* \\ ${ }^{1}$ Department of Physical Education, Anhui University of Traditional Chinese Medicine, Hefei, China \\ ${ }^{2}$ Department of Physical Education, Fuyang Teachers College, Fuyang, China \\ ${ }^{3}$ Faculty of Chinese Medicine, Macau University of Science and Technology, Macau, China \\ Email: *496664517@qq.com, ${ }^{*}$ cscheng@must.edu.mo
}

Received 24 February 2016; accepted 27 March 2016; published 30 March 2016

Copyright (C) 2016 by authors and Scientific Research Publishing Inc.

This work is licensed under the Creative Commons Attribution International License (CC BY).

http://creativecommons.org/licenses/by/4.0/

(c) (7) Open Access

\begin{abstract}
Our investigations indicate that Wuqinxi in Singapore has now been studied and is mainly promoted through performing exercises, exchanges and competition. The Singapore government has regarded for Qigong for many years, and thus helps Chinese Wuqinxi developed a successful foundation in Singapore. Besides, this research promptes that the Wuqinxi practitioners are mostly women who are around the age of 20 - 40 and their occupations are mainly workman, civil servants and self-employment. Most of them learn Wuqinxi through Wuqinxi exercises classes or in the Wushu clubs. In Singapore, people improve their health through learning and practice Wuqinxi which cater the demand for municipal and have been promoted and advocated by levels of government departments. It is encouraging that the data indicate that $75.7 \%$ of the Wuqinxi practitioners practice 1 - 3 times per week. The data seem to indicate that China Wuqinxi will well developed in Singapore. However, The main obstacles affecting the promotion of Wuqinxi in Singapore are the lack of professional action coaches and theoretical education of sports action.
\end{abstract}

\section{Keywords}

Wuqinxi, Singapore, Qigong, Promotion

\section{Introduction}

Since the General Administration of Sport of China established the Qigong Administration Centre on $30^{\text {th }}$ April, 2015, it helped the Qigong to expand further internationally [1] [2]. In China, Qigong is so popular among the

${ }^{*}$ Corresponding authors.

How to cite this paper: Zhang, B., Ru, L., Lin, H., Liang, J., Lao, C.C., Peng, D.Y. and Cheng, C.S. (2016) Dissemination and Development of Wuqinxi in Singapore. Open Journal of Social Sciences, 4, 264-271.

http://dx.doi.org/10.4236/jss.2016.43033 
baby boomers (elderly people), but no one notices the importance of teaching the new generation about the traditional exercise called "Qigong”; this impacts on the popularity and the development of Qigong [3] [4].

Wuqinxi is a set of nation-typical Qigong, made by great physician Huatuo in Donghan Dynasty. According to the ancient "Daoyin" and "Tuna" (Ancient breathing exercise of Taoism), Huatuo carefully observed the activity characters of tiger, deer, bear, ape and bird 5 animals, and composed the set of actions integrated with the combination of human body functions, and the biological clock [5]-[7]. Wuqinxi can calm the brain, relax the body, and keep balance of physical and psychological, through regulating the breath and a set of slowly body movement. Moreover, it can directly act on the central nerve system and vegetative nerve system [8]. Those reduced the stimulation of bad mood on brain and then maintained relative stabilization of internal environment of human body [9]. Emphasizing on the concept of integrity is the second feature of Wuqinxi [10]. Studies reported that the functions of Wuqinxi were not development of partial body functions and treatments of certain disease but comprehensive exercise by recuperating body and psychology [11]. Simplicity and popularity are the third of feature of Wuqinxi [12]. Practitioners exercising Wuqinxi do not depend on place, exercise equipment, whatever sex or different age levels [13]. The practitioners can select different routine of Wuqinxi according to one's requires and conditions.

Qigong, a diverse exercise, has been launched as the $62^{\text {th }}$ Sport in China by the Chinese General Administration of Sport, which is simple, soft and relax, and the playing space are not restrictive [14]. Therefore, Qigong strongly builds up in the public and increases the popularity of Qigong, gaining more practitioners [15]. Qigong is currently promoted overseas through many different ways, such as conferences, health lectures, performances, training class, etc. [16] [17]. However, there is not a concern about the ways of promoting Qigong worldwide. Therefore, this research taking Wuqingxi as an example, we investigated the promotion of Wuqinxi in Singapore and analysed the development context, positive factors and the prospects, which would help the success of the global Qigong’s promotion.

\section{Methods}

\subsection{Literature}

Through studying the theses of CNKI, WANFANG, VIP and other large periodical databases, and collecting all the data and information of Qigong since the year of 2000, it offers the theoretical basis for the aim of this report.

\subsection{Interviews}

The studies were taken by using phone calling and face-to-face interviews relating to the concept and the purpose of this report, which helps to create the questionnaire in this paper. Through interviews with the Qigong promotion organisation managers and coaches, it significantly provided some professional information and suggestions about the project of Qigong promotion in Singapore, including different types and forms of the project, as well as the event venues. In addition, the interviews also help people to understand the current situation of Qigong promotion in Singapore.

\subsection{Questionnaire}

The questionnaire was made during the annual exchange in Singapore and provided as a supplement in the end of the manuscript, the mainly contents include interviewees information, expertise-understanding of Wuqinxi, learning and practicing situations, health recovery etc. And finished by partners and students, 500 questionnaires have been handed out. However, there are only $94.6 \%$ of the total 500 questionnaires received in succession. Excluding 16 invalid questionnaires, there is a $96.6 \%$ of effective rate which indicates that the success of the questionnaire workout. Before the beginning of the investigation, the contents' validity of the questionnaire was evaluated by 9 traditional sports experts who serve on higher institute of physical education colleges for more than 10 years. Furthermore the reliability analysis of the questionnaire data obtained in this study was analysed by SPSS 17.0 and showed the Cologne Bach $\alpha$ reliability coefficient is 0.697 .

\subsection{Entry Criteria to Participants}

Respondents in this investigation, shall be held in Singapore identity-card or proof of their long-term stay. 
Evenly distributed gender is one of the scientific designs in this study, and an effective respondent is including 59.1\% females and $40.9 \%$ males. The age distribution of the practitioners has been showed in following Figure 1. In addition, the experts interviewed in this research must have more than 3 years' experiences in the Chinese martial arts education or related communication in Singapore.

\subsection{Mathematical Statistical Analysis Method}

Collection of literatures collated and analysed firstly and the investigation results of the survey statistics processed by software including Excel, SPSS17.0. The survey data were collected and analysed with Excel 2007, and related statistical analyses in this paper were operated with the SPSS 17.0 including the Cologne Bach $\alpha$, ANOVA, T-test etc.

\section{Results and Analysis}

\subsection{The Mode of Wuqinxi Development in Singapore}

According to the Table 1, it proved that the development of Wuqinxi in Singapore is successfully made by inviting Chinese Qigong organisation to do Qigong training, as well as the Qigong communions and contest. Those significantly increased the popularity of Qigong in Singapore.

\subsection{The Occupation of Wuqinxi Practitioners in Singapore}

As shown in Table 2, Workman, civil servants, self-employments, and students are the major components of the Wuqinxi practitioners. Singapore is a really good place to live, work and relaxes, and in which there are so many Singaporeans-born Chinese. Singapore government has a positive attitude at people, quality of life. Therefore, as a traditional fitness program in china, Wuqinxi has attracted people from the all walks of life, and Qigong has also received the support of government departments.

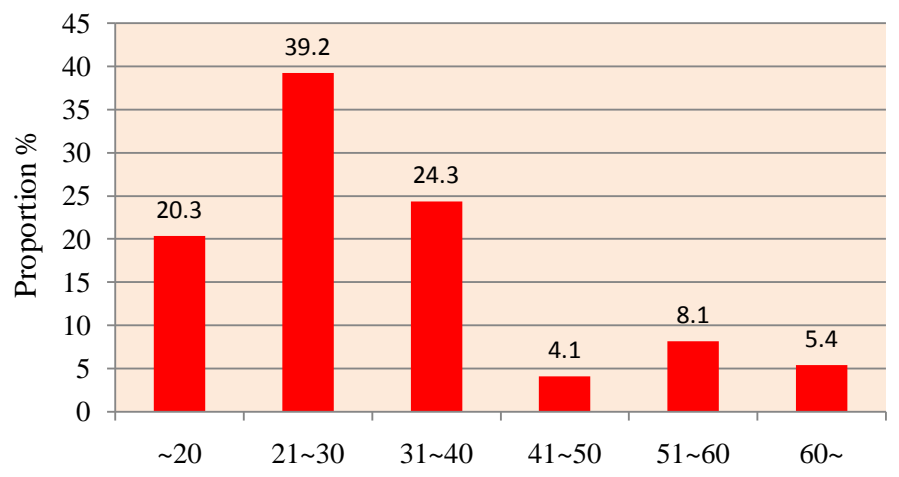

Age contribution of the Wuqinxi practitioners (Years)

Figure 1. The age distribution of the Wuqinxi practitioners in Singapore.

Table 1. The mode of wuqinxi promotion in Singapore $(\mathrm{N}=457)$.

\begin{tabular}{cccccc}
\hline Mode & Speech & Class & Performance/Communion & Contest & Media Publicity \\
\hline Number of people & 40 & 51 & 225 & 114 & 27 \\
Rate (\%) & 8.8 & 11.2 & 49.2 & 24.9 & 5.9 \\
\hline
\end{tabular}

Table 2. The occupation of Wuqinxi practitioners in Singapore $(\mathrm{N}=457)$.

\begin{tabular}{ccccccc}
\hline Occupation & Student & Public servant & Workman & Self-employment & Retiree & Other \\
\hline Number & 61 & 116 & 146 & 104 & 18 \\
Rate (\%) & 12.2 & 23.2 & 29.2 & 20.8 & 3.6 \\
\hline
\end{tabular}




\subsection{Learning and Teaching of Wuqinxi in Singapore}

As shown in Table 3, the investigation of the understanding of Singapore Wuqinxi practitioners, it indicated that Wuqinxi practitioners have few understanding of the advantage of Wuqinxi fitness functions, such as fitness mechanisms and intrinsic applications. Theoretical knowledges can guide the practice to be a set of scientific activity, and so a comprehensive understanding of the theoretical knowledge of Wuqinxi clearly and grasping of the own perception of the sport have become a positive role in promoting of Wuqinxi in Singapore. For example, through understanding the theoretical knowledge of Wuqinxi, practitioners can effective improve different parts of their bodies. All of the interview records indicated that the Wuqinxi practitioners can make practicing moves correctly, but most of them do not know the advantage of each actions of Wuqinxi. But, there are also some of the practitioners responded lower level definitions of Wuqinxi, such as just exercise or sweating. Therefore, this paper suggested that theoretical knowledge should be firstly promoted in Singapore. Qigong Wuqinxi, came from ancient Chinese traditional culture, the promotion development of Quqinxi in Singapore should strengthen the theoretical knowledge to the practitioners, which would help practitioners more understanding the value of fitness culture and health of Qigong "Wuqinxi”, and balance the theory and practice with their self. Wuqinxi is not only mimicking the action of five animals, but also includes five animal habits and theoretical knowledges. These studies also indicated that theoretical learning for practitioners can make a real understanding of fitness and cultural values of Qigong.

\subsection{The Wuqinxi Practitioners' Channels of Learning Wuqinxi in Singapore}

The Data of Table 4 shows that more than a half of the practitioners learn Quqinxi in their professional classroom learning. It showed that the construction of Wuqinxi courses in Singapore had a certain scale, which is closely related to the support of the Singapore government to carry out Health Qigong. By visiting the Wuqinxi classes in Singapore, those classes includes more mature and rational way which are based on the interest of students, different levels of understanding of Wuqinxi and physical fitness index, and then divided people into 3 level classes, including high, middle and junior. This systematic teaching method caused significant effects of high standard of receiving the information. However, Wuqinxi teachers in the classes do not teach the participants with the theoretical knowledge of Wuqinxi. Instead, the tutors were only focusing on the practice, correcting the poses for the students. In addition, through interviews with the students, we learned that the students are losing interests on Wuqinxi due to those "step by step" and the tutors are only focus on their poses.

Training classes are the main approach to Singaporeans to learn Wuqinxi. Wherein, the degree of specialization coaches have directly impacted and sustainability constrained the Qigong in Singapore. A comprehensive improvement including specialized technical movements and theoretical knowledge of Qigong was trained in 2013, Singapore has trained the opening ceremony, which provides and developed a good coaching specialization. Therefore, we thought that the coaches training classes improved the Wuqinxi promotion development and increased the popularity of Qigong in Singapore. Through the Wuqinxi poses’ presentation of coaches, theoretical knowledge explaining and repeat demonstration of key exercise, students can effectively gain the knowledge of Qigong. In the process coaches’ specialization also has been improved, which is advantage to Singapore Qigong practitioners services and would promote the health Qigong in Singapore indirectly.

Table 3. The situation of Wuqinxi practitioners understanding Wuqinxi theoretical knowledge in Singapore $(\mathrm{N}=457)$.

\begin{tabular}{rccccc} 
& Better understanding & Understanding & General understanding & Few understanding & Not understanding \\
\hline Number & 46 & 104 & 202 & 85 & 20 \\
Rate (\%) & 10.1 & 22.8 & 44.2 & 18.6 & 4.4 \\
\hline
\end{tabular}

Table 4. The Wuqinxi practitioners' channels of learning Wuqinxi in Singapore $(\mathrm{N}=457)$.

\begin{tabular}{ccccc}
\hline Channel & Square & Courses & Unit & Kisfolk and friends \\
\hline Number & 34 & 229 & 47 & 128 \\
Rate (\%) & 7.4 & 50.1 & 10.3 & 28 \\
\hline
\end{tabular}




\subsection{Practice Purpose of Wuqinxi}

Wuqinxi as a martial arts class sport, which naturally includes sport basic attributes that enhance physical fitness, improve their health, and this is also the essence of Wuqinxi. However as the Table 5 showing, only $54.2 \%$ of practitioners considered their practice purposes as the sport basic attributes of Wuqinxi movement. Through interviews investigation, the main reason for that insure of ignoring of the significance Wuqinxi fitness: 1) Most people don't understand the fitness function of Wuqinxi; 2) Social needs of some people are higher than their fitness needs; 3) Some people learn it because of their curiosity. In conclusion, potential practitioners and learners of Wuqinxi are lack of the professional fitness awareness so that their untagged purpose cannot promote them to do well. So we suggested that the government or related associations should intensify its work on social communication for Wuqinxi, and similarly, the actual health significance of each action also need to be clear.

\subsection{The Exercise Frequency of Wuqinxi in Singapore}

The data in Table 6 showed that more than $70 \%$ of Wuqinxi practitioners in Singapore participate in Wuqinxi exercise at least 3 times per week, which is closely related with the age and occupation of Singaporeans who practice the Qigong Wuqinxi; The data showed in Table 2 described occupation and age of the practitioners which showed that main participant is between 20 years old to 40 years olds; the occupational component of Singapore Wuqinxi practitioners are workers, students from institutions, individual professionals. These practitioners are in learning, entrepreneurship and working stage and effectively into the cause of Wuqinxi exercises but have no enough time to practice their own fitness program, and turn that they led to a really few number of times per week to participate the Wuqinxi exercise as shown in Table 6.

\subsection{Satisfaction Survey of Wuqinxi Practitioners}

In the internationalization process of Qigong Wuqinxi, we have attempted to attract more Wuqinxi practiser through the efforts of practitioners and the important approach was participating in active Qigong exercise in the past. Besides, we also expected the propagation of our Chinese culture can promote the development of Qigong Wuqinxi. However, current survey data have not yet been reported to indicate the satisfaction of Wuqinxi practitioners. So in this paper the survey results showed that promotion of Health Qigong Wuqinxiin Singapore have basically meted the needs of practitioners as shown in Table 7 and 67\% of Wuqinxi practitioners practiced it with no objectionable, $30 \%$ of them expressed their satisfaction. But it does not mean that Wuqinxi promotion in Singapore has made great achievements, which just proved that Wuqinxi in Singapore have a higher demand benefiting from propagation and development of Wuqinxi in the past 10 years. Qigong promotion in the international arena is a dynamic process of long-term practice. With the improvement of the social development and

Table 5. The purpose of Wuqinxi practitioner exercise Wuqinx in Singapore $(\mathrm{N}=457)$.

\begin{tabular}{cccccc}
\hline Purpose & Fitness & Make more friends & Entertainment & Following kisflok and friends & Other \\
\hline Number & 248 & 76 & 63 & 44 & 25 \\
Rate (\%) & 54.2 & 16.7 & 13.9 & 9.7 & 5.6 \\
\hline
\end{tabular}

Table 6. The times of Wuqinxi practitioners exercise Wuqinxi in Singapore.

\begin{tabular}{cccccc}
\hline Times/week & 1 to 2 times & 2 to 3 times & 3 to 4 times & 4 to 5 times & 5 or more \\
\hline Number & 194 & 152 & 55 & 21 \\
Rate (\%) & 42.4 & 33.3 & 12.1 & 7.6 \\
\hline
\end{tabular}

Table 7. The satisfaction of Wuqinxi practitioners in Singapore $(\mathrm{N}=457)$.

\begin{tabular}{cccccc} 
& Very satisfied & satisfied & General & Dissatisfied & Very dissatisfied \\
\hline Number & 85 & 52 & 163 & 137 & 20 \\
Rate (\%) & 18.6 & 11.4 & 35.7 & 30 & 4.3 \\
\hline
\end{tabular}


living standards, practitioners choose fitness exercises for their daily plan is constantly changing. Therefore, Qigong in international communication process should be based on the individual needs of practitioners in different seasons, different weather conditions, and different venues.

\subsection{The Evaluation of Qigong Practitioners in Singapore}

As shown in Table 8, the results showed that 31.1\% of Qigong practitioners believed that Qigong is very good, and they can enjoy in Wuqinxi exercises; $42 \%$ of them said the Qigong exercise is good. Survey showed with interviews, indicated that more of the practitioners agreed with the efficacy of Wuqinxi; but few of them like Tai chi more and thought Wuqinxi is not a universal practicing sport. Overall analysed the Table 8, it indicated that the vast majority of practitioners after Qigong exercises, and Qigong Wuqinxi is a very good representation of sport exercise, these people have accounted to $71.1 \%$ of practitioners.

\subsection{The Favorable Conditions to Wuqinxi in Singapore}

\subsubsection{The Bilateral Friendly Exchanges of Political and Economic}

The frequent trade of China and Singapore is the cornerstone to development of Qigong, since Singapore is a smaller island, many resources need to be imported, China is one of the major importing countries for Singapore, of course, also included many industrial products to be imported, China has more than 5200 companies have investments in Singapore. Although sometimes not friendly relationship between Singapore and China, but the political and economic exchanges have been very frequent, so the frequent contact is the cultural communication foundation. In this exchange process, more than 100 million people in and out of Singapore per year. All levels of Chinese government sent numerousmartial arts delegations to Singapore in the past 30 years.

\subsubsection{The Important Roles of the Local Chinese Community}

Singapore is a multi-ethnic country, mainly including Han Chinese, Indian, Malay, Eurasian family etc. Wherein, the Han Chinese is more than $89 \%$ of the total population and nearly 50 million people are the overwhelming majority of Chinese and Chinese descendants, and almost all these Han Chinese came from Fujian and Chaozhou, Cantonese, Hakka. Moreover, Singaporeans itself, most of them have Chinese people's blood, so Chinese culture is also an important part of Singapore traditional culture. Beside, Singapore's official language is Chinese, which also contributed with a great extent for Chinese Wushu, especially for the dissemination and development of Qigong Wuqinxi in Singapore.

\section{Discussions}

There are so many forms of gymnastically qigong in China including Tai-chi, Wuqinxi, Wing Chun, Yi Jin Jing and so on [18] [19], Which changed and regulated the Chinese people's daily exercise and fitness mode for thousands of years. However, the scientific connotations have not yet been revealed until now by all the mankind [20] [21]. A large number of rehabilitation-related scientific studies have been reported in recent years [11], Singapore, a young country, a large number Chinese living in, is being busted with a kind of qigong practice social atmosphere [22] [23]. In such an atmosphere, strict standards and scientific researchers began with many Chinese people's participations in Singapore. Our research team, composed with 15 senior title physical teachers or martial art instructors, 14 people consisting of two Wushu team are also instructed by us. Years of teaching and referee experiences promoted a close exchange with Singapore's counterparts, and in turn made the investigation carried out so successfully. Compared with the similar surveys all over the word, we believed that this survey will greatly promote Chinese Qigong scientific training in Singapore and expand its influence in the world. Moreover, this means of the investigation will guide investigating other wushu sports in the other countries.

Table 8. The evaluation of Wuqinxi practitioners of Fitness Wuqinxi in Singapore $(\mathrm{N}=457)$.

\begin{tabular}{cccccc}
\hline Evaluation & Very good & Good & General & Bad & 31 \\
\hline Number & 142 & 192 & 81 & 11 \\
Rate (\%) & 31.1 & 42.0 & 17.7 & 6.8 & 2.4 \\
\hline
\end{tabular}




\section{Conclusion}

Qigong Wuqinxi has been concerned by all levels of governments in Singapore, which is the mainly foundation for the promotion of Qigong Wuqinxi in Singapore. The main form of promotion of the Wuqinxiin Singapore is performing exercises, exchange and competition. But Singaporeans have been proved lacking of the overall Wuqinxi theoretical knowledge for fitness and learning media of theoretical knowledge of Qigong is relatively narrow; our investigations showed that they obtained their mainly fitness theoretical and Chinese culture through the new media. Our research suggests that Wuqinxi training institutions is looking forward to a further improvement; and Wuqinxi should be teaching combined to traditional martial arts just like the Tai Chi; Enhanced Wuqinxi teaching staff construction, increased the intensity of training theoretical knowledge of Wuqinxi, scientific media campaigns are also important to Wuqinxi in Singapore.

\section{Acknowledgements}

The authors would like to thank the contract grant sponsors of Education Department, Anhui Province, China. Humanities and social sciences research: SK2014A284 and Sports Social Science Research of Anhui Province ASS2016207.

\section{References}

[1] Yu, D.H., Chen, W.H., Zhang, S.Z., et al. (2003) Compilation of New Wuqinxi Style and Its Experimental Effect. Journal of Shanghai Physical Education Institute, 27, 55-58.

[2] Zhao, B.K. (2013) The Study of the Promotion of Health Qigong and Its Present Situation and Improvement. Contemporary Sports Technology, 3, 104, 106.

[3] Kuang, H.L. and Liu, H.C. (2010) Analysis on Development Trend and Condition of Health Qigong Activity Stations Management in Community. Bulletin of Sport Science and Technology, 18, 88-90.

[4] Yu, D.H. and Wang, L. (2009) Promotion Strategies of Newly Compiled Health Qigong. Journal of Institute of Physical Education, 43, 65-69.

[5] Li, X.F. (2008) Research on the Development of Our Health Qigong Associations in Present Stage. Beijing Sport University, 25-158.

[6] Zhong, L.L. and Yu, D.H. (2007) Research on the Construction of Health Qigong Promotion Mode. The 8th National Convention on Sport Science of China.

[7] Wang, L. (2009) Research on Popularization Strategy of Health Qigong. Shanghai University of Sport, 1-88.

[8] Fan, Y.W. and Gao, H.Y. (2013) Analysis on Current Situation of the Spread of Health Qigong among the Oversea Chinese Community. Southeast Asian Studies, 3, 97-102.

[9] Zhang, Y.Y., Wang, L. and Yu, D.H. (2008) Present Situation for Promotion and Popularization of Health Qigong. Journal of Shandong Institute of Physical Education and Sports, 24, 29-32.

[10] Wei, X., Xu, A., Yin, Y., et al. (2015) The Potential Effect of Wuqinxi Exercise for Primary Osteoporosis: A Systematic Review and Meta-Analysis. Maturitas, 82, 346-354. http://dx.doi.org/10.1016/j.maturitas.2015.08.013

[11] Tong, S.M., Yu, D.H. and Wang, M.J. (2011) Current Situation of Popularizing for Fitness Qigong in Oversea. Journal of Shadong Institute of Physical Education and Sports, 27, 24-28.

[12] Zhang, B., Cheng, C.S. and Guo, Y.P. (2013) Research Overview of Wuqinxi on Human Performance Intervention. Journal of Hubei University of Economics, 10, 41-46.

[13] Yan, L. (2014) Health Qigong Marketing Strategy: A Case Study of Wuqinxi. Wushu Science, $11,37$.

[14] Lei, F., Yan, J.T. and Sun, K.X. (2013) Progress on Chinese Traditional Regimen of Wuqinxi Exercise. China Journal of Traditional Chinese Medicine and Pharmacy, 3, 73.

[15] Ru, J.J. and Wang, M.D. (2013) On the Effects Research Status of Wuqinxi Exercise and Tai Chi on the Elderly Health. Wushu Science, 1, 31.

[16] Bin, X. (2015) Study on the Impact of "Health Qigong.WuQinxi” on the Physical Health of the University Students. Atlantis Press, 530-536.

[17] Cui, Z. (2012) Research on Ownership of the Event Groups and Training Methods of the Health Qigong Wuqinxi. Sports Forum, 5, 34.

[18] Green, T.A. (2001) Martial Arts of the World. Santa Barbara, California, 758, 13-17.

[19] Yang, J. and Weng, M. (1999) Taijiquan, Classical Yang Style. YMAA Publication Center, Canada, 1-54. 
[20] Zheng, Z. (2012) The Research Progress of Fitness Qigong Wuqinxi in the Past Five Years. Nei Mongol Journal of Traditional Chinese Medicine, 18, 114.

[21] Qiu, F.B. (2004) Effects of Yoga to the Functions of Psychology and Physiology. Journal of Shandong Physical Education Institute, 5, 20.

[22] Lian, W.M., Gan, G.L., Pin, C.H., et al. (1999) Correlates of Leisure-Time Physical Activity in an Elderly Population in Singapore. American Journal of Public Health, 89, 1578-1580. http://dx.doi.org/10.2105/AJPH.89.10.1578

[23] Pan, L.L., Liu, X.H., Gong, Q.H., et al. (2012) Role of Cystathionine $\gamma$-Lyase/Hydrogen Sulfide Pathway in Cardiovascular Disease: A Novel Therapeutic Strategy. Antioxidants \& Redox Signaling, 17, 106-118. http://dx.doi.org/10.1089/ars.2011.4349 